\title{
The Fraction of the Segment in Three Parts
}

\author{
Enfer Diez \\ Institute polytechnic, leon, Spain \\ *Corresponding Author: emigarcia4@gmail.com
}

Copyright (C) 2013 Horizon Research Publishing All rights reserved.

\begin{abstract}
In this work I present the method to divide with rule and the compass any segment given in three parts and also, the polynomials of degree one for the construction of the angle with rule and the compass: $b^{2} x-2 x a^{2}+2 a^{2}=0$ (1) for any angle that belongs to an isosceles triangle. ( $\left.b^{\prime}\right)^{2} x$ $-2 x a^{2}+2(a+n)^{2}=0 \quad$ (2) for any triangle which sum of two of his angles is minor to $90^{\circ}$, always $(2 \mathrm{a}>\mathrm{b}) ;(\mathrm{x}>1)$; $\mathrm{x}=(2 ; 3 ; 4 ; 5 ; 6) .\left(\mathrm{b}_{\mathrm{x}}\right)^{2} \mathrm{p}_{1}-2 \mathrm{a}^{2} \mathrm{p}_{1}+2 \mathrm{a}^{2} \mathrm{p}=0$

(p) prime number fixed, and $\mathrm{x}=\left(\mathrm{p}_{1} ; \mathrm{p}_{2} ; \mathrm{p}_{3} ; \ldots \ldots \ldots . \mathrm{p}_{\mathrm{k}}\right)$; always $\left(\mathrm{p}<\mathrm{p}_{1}<\mathrm{p}_{2}<\ldots \ldots\right)$.
\end{abstract}

Keywords New Polynomial, Fraction Third with the Rule and the Compass

\section{Introduction}

We all know the minimal degree of a polynomial for any length construable with rule and the compass is a power of two. And also that can be constructed by the process origami or well by the rule marked with Arquimedes and Pergamo's Apolino. Hipias's trisectriz in a curve that we know that is projection ids not possible.

The impossibility for the trisection of an angle is based on the need to have the cube root of a complex number.

Since well this is avoided applying the following process for the fraction $(y / 3)$ in any segment.

\section{My Results}

We take the segment (AB) we plan his square (Figure 1) and in him, his respective diagonals, later we divide the segment $(\mathrm{AB})$ by the half and from this one point we plan two lines to the opposite vertex, observe that they insert respectively in the diagonals; if now from each of these points we plan perpendicular lines $(\mathrm{AB})$ we will have in $(\mathrm{AB})$ three equal segments $(\mathrm{c} ; \mathrm{d} ; \mathrm{a})$.

Demonstration:

For the (Figure 1) we have that:

$$
\begin{gathered}
a+n \sqrt{ } 2=A B \\
(n+m) \sqrt{ } 2=A B
\end{gathered}
$$

therefore:

$a+n \sqrt{ } 2=(n+m) \sqrt{2}$; it implies that: $(m \sqrt{2}=a)$ and consequently.

$(a+d+c) \sqrt{2}=2 n+2 m ;$ as $(m=n / 2)$ we have.

$(a+d+c) \sqrt{ } 2=3 n$

It is to say: $A B \sqrt{2}=3 n$; with which $(A B=3 n / \sqrt{2}$ we have demonstrated that $(a=d=c)$

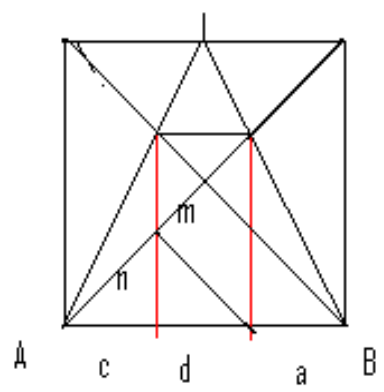

Figure 1. Fraction of the segment $\mathrm{AB}$ in three.

Done the demonstration we happen to construct with rule and the compass a given angle; if we have the triangle (oba) (Figure 2) we take of reference the angle of the vertex (o) and the we increase three times.

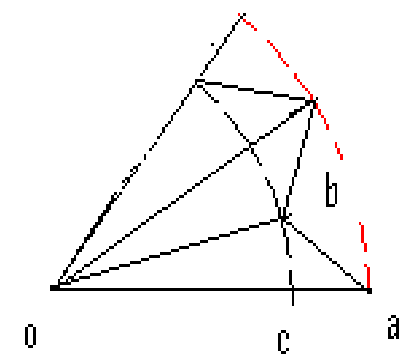

Figure 2. Three times increase the angle of the triangle (oba)

His process is the following one, we plan two arches of respective radiuses $(0 \mathrm{c})$ and (oa) later with the compass, project the segment (ba) from the vertex (b) in the exterior arch and, from this point we return to project it in the interior arch, in this instant is triplicate the initial angle ( we have formed a triangle that contains three times the triangle (oba)).

Now we happen to the inverse process, that is to say, to give of an angle limited by the sides (oa) (Figure 3) to do the 
trisection of the angle with the rule and the compass; the first we plan the rope (aa) and to divide her in three equal parts we apply the process of, to divide an segment in three.

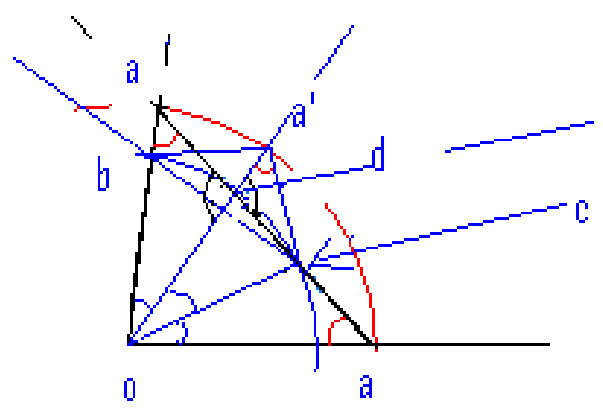

Figure 3. fraction of an angle with rule and the compass.

Later with the compass we project the segment $(\mathrm{ac}=(\mathrm{aa}) / 3)$ in the same form as we have done in the (Figure 2); we observe that we have completed the whole angle that form the lines (oa), using three times the triangle (oac) or what is the same thing, three times the angle that form the lines (oa) and (oc).

To demonstrate that the triangle (oca) is equal to (oca') is simple because, $\left(a^{\prime} c=a c\right)$ and in turn $\left(\mathrm{oa}^{\prime}=\mathrm{ao}\right)$ and $(\mathrm{oc})$ is common to both; now well the third one that for his evidence in the construction we say that it is equal to both. His corroboration is in the angle that form the intersection of the lines (aa) with (oa') vertex (d), are equal and belong to the triangles (a'dc) and (ado) both have jointly the angle of the vertex (a); if two triangle have two equal angles the third party also it is and, therefore they are similar or be equal. In turn the triangle (a'dc) is similar to the (a'co) and consequently the angle form the lines (cd) and ( $\left.a^{\prime} c\right)$ is equal to the angle is between the lines (a'o) and (co); this checks that the three angles of the vertex (o) are equal. This is corroborated by the polynomials.

Any angle is divided if it fulfils the polynomial: $\mathrm{b} 2 \mathrm{x}-$ $2 \mathrm{a} 2 \mathrm{x}+2(\mathrm{a}+\mathrm{n}) 2=0$; this angle corresponds to a triangle which sum of two of his angles is minor to $90^{\circ}$; always ( $2 \mathrm{a}>$ b). The only value that is not possible is $(x=0 ; x=1)$.Or it is fulfils the polynomial: $\mathrm{b} 2 \mathrm{x}-2 \mathrm{a} 2 \mathrm{x}+2 \mathrm{ax}=0$

Later we happen to analyze the polynomial "(1)": if we leave the value of (x) constant and change the values of (a) of random form we will have always the same angle belonging to an isosceles triangle (similar) and, if we leave of (x) constant and increase one of the values of (a) we continue with the same angle and, have changed to a triangle oblicuangulo (polynomial 2); nevertheless if we change (x) and leave two of his coefficients constant then, the angle is modified. $\left(\mathrm{b}<\mathrm{b}^{\prime}<\mathrm{b}_{1}<\mathrm{b}_{2}\right)$.

$$
\begin{gathered}
b^{2} x-2 a^{2} x+2 a^{2}=0 \\
\left(b^{\prime}\right)^{2} x-2(a+n)^{2} x+2(a+n)^{2}=0 \\
\left(b_{1}\right)^{2} x-2 a^{2} x+2(a+n)^{2}=0 \\
\left(b_{2}\right)^{2} x^{\prime}-2 a^{2} x^{\prime}+2 a^{2}=0
\end{gathered}
$$

Another relevant tact is the following one: if we have values of $(b ; a$; $x)$ they fulfil the polynomial (2) and, we multiply by $(\mathrm{m})$ we have: (2) $\mathrm{m}[\mathrm{b} 2 \mathrm{x}-2 \mathrm{a} 2 \mathrm{x}+2(\mathrm{a}+\mathrm{n}) 2]=0$; this is equivalent to the arithmetical operation of the sum of (m) times an angle.

It is to say for the arithmetic: $b 2 \mathrm{mx}-2 \mathrm{a} 2 \mathrm{mx}+2 \mathrm{~m}(\mathrm{a}+\mathrm{n}) 2=$ m.0; nevertheless in geometry $(b ; a ; a+n)$ they are three segments closed by three angles (geometric figures) and therefore we will need a new polynomial where his root is $(0)$, to define the polynomial we have two options, change three coefficients and respect the value of the variable (x) or will, increase the variable and to respect two of the coefficients, that is to say.

The equation $(\mathrm{mb}) 2 \mathrm{x}-2(\mathrm{am}) 2 \mathrm{x}+2[\mathrm{~m}(\mathrm{a}+\mathrm{n})] 2$; this is correct because we have same angle that in the polynomial (2); since we have continued with the same value of the variable (x); obviously if we divide the polynomial by ( $\mathrm{m} 2)$ we have of new the polynomial (2).

Now well if we say that $\left(\mathrm{mx}=\mathrm{x}^{\prime}\right)$ is to say to modify the variable we have that.

$$
\mathrm{b} 2 \mathrm{x}^{\prime}-2 \mathrm{a} 2 \mathrm{x}^{\prime}+2[\mathrm{~m}(\mathrm{a}+\mathrm{n})] 2=0
$$

Of this polynomial we know that, if we respect two of the coefficients and we increase the value (x), rhen we have increased the angle given in (m) times; this is the same thing that we have done with the geometric process of the rule and the compass, (Figure 3).

Finally I indicate because of these polynomials. His origin is in $\mathrm{T}$. of the Cosine.

$$
b^{2}=2 a^{2}-2 a^{2}(\cos (n))=2 a^{2}(1-\cos (n))
$$

all the value of the cosine is determined in the isosceles triangle and in turn, any value of the cosine is minor to the unit $(\cos (n)<1)$ consequently also $(1 / x=\cos (n))$; with which we say that.

$$
1-\cos (n)=1-1 / x=(x-1) / x
$$

By the equation of the T. cosine say that.

$$
\left(b^{2}\right) / 2 a^{2}=(x-1) / x
$$

Therefore.

$$
\begin{array}{r}
b^{2} \mathrm{x}=2 \mathrm{a}^{2}(\mathrm{x}-1) \\
\mathrm{b}^{2} \mathrm{x}-2 \mathrm{a}^{2} \mathrm{x}+2 \mathrm{a}^{2}=0
\end{array}
$$

Also we will have values for the cosine with the relation of prime numbers in the form of $(3)[\mathrm{p} / \mathrm{p} 1) ; \mathrm{p} / \mathrm{p} 2 ;<1] ; \mathrm{p}<\mathrm{p} 1$ $<$ p2 $<$; for this relation there is defined the polynomial (3) that in turn, also we determine the polynomial (3) replacing in the polynomial (1) the value of $(x)$ by the relation $(\mathrm{p} / \mathrm{p} 1)$.

\section{Conclusion}

The arithmetical sum of the angle is equivalent to the arithmetical sum of the side opposite to the above mentioned angle. 


\section{REFERENTES}

[1] D. Brill, Brilliant origami, Japan publications, Tokio (2001)

[2] Richelot Friecdrid Julios, de resolutione algebraica aequationis $\mathrm{x}^{257}=1$, sive de divisione circuli por bisectionen anguli septies repetitam in partes 257 inter se aequales comentario coronete, Journal für die Reine und Angewandte Mathematik 9, pp1-26, 209-230, 337-358 (1832)

[3] T. Fuse, unit origami, multidimensional transformation, Japan Publication (2000)
[4] G. F. Gauss, disquisiciones arithmeticae, Werke Eriter Band (1863)

[5] C. G. Gibson, elementary geometry of differentiable curves, Cambridge University Press (2001)

[6] A. Gray, modern differential geometry of curves and surfaces with mathematica, CRC Press (1998)

[7] Wantzel M. L; recherches sur los moyens de reconnoitre si un problème de Gèmètrie peut se rèsoudre avec la règle et le compas, Journal de Mathematiquies 1; pp 336-372, (1837 